\title{
La radioterapia en el tratamiento de las metástasis coroideas y cerebrales simultáneas
}

\author{
C. CONILL, S. JORCANO, I. PLANAS, J. MARRUECOS, F. CASAS, J. R. FONTENLA ${ }^{1}$ \\ Servicio de Oncología Radioterápica y ${ }^{2}$ Servicio de Oftalmología. Hospital Clínic. Institut \\ d'Investigacions Biomèdiques August Pi i Sunyer (IDIBAPS). Universidad de Barcelona. \\ Barcelona
}

\begin{abstract}
RESUMEN
Las metástasis coroideas de cáncer de pulmón pueden presentarse como primera manifestación clínica de extensión metastásica de la enfermedad aunque suelen coexistir con al menos dos localizaciones metastásicas. La sintomatología más frecuente es la disminución de la agudeza visual. Sin embargo, hasta un $20 \%$ de las metástasis cerebrales también pueden presentar alteraciones visuales, por lo que debe realizarse el diagnóstico diferencial entre metástasis cerebral y/o coroidea.

Presentamos un caso de una paciente diagnosticada de adenocarcinoma de pulmón que a raíz de una disminución de la agudeza visual fue diagnosticada de metástasis coroideas y cerebrales simultáneas. Fue tratada con irradiación holocraneal (DT 20Gy/5fracciones) con una mejoría significativa de la agudeza visual. Este caso ilustra que aunque la expectancia de vida de los pacientes con cáncer de pulmón metastático es corta, un diagnóstico y tratamiento adecuado puede mejorar la calidad de vida de estos pacientes.
\end{abstract}

PALABRAS CLAVE: Metástasis coroideas. Metástasis cerebrales. Cáncer de pulmón. Radioterapia.
RADIATION THERAPY IN SIMULTANEOUS CHOROIDAL AND BRAIN METASTASES

Conill C, Jorcano S, Planas I, Marruecos J, Casas F, Fontenla JR. La radioterapia en el tratamiento de las metástasis coroideas y cerebrales simultáneas. An Med Interna (Madrid) 2005; 22: 431-433.

KEY WORDS: Choroidal metastasis. Brain metastasis. Lung cancer. Ra-diotherapy.

\section{INTRODUCCIÓN}

Las metástasis intraoculares representan la mayor parte de los tumores malignos del ojo con una incidencia en autopsias del 12\%(1). Los tumores primarios más frecuentes son mama (62\%) y pulmón (26\%), siendo la primera manifestación clínica de su enfermedad en el 18\% de los pacientes con cáncer de pulmón (2). En estudios clínicos de programas de detección en pacientes con cáncer de mama se han descrito incidencias del 5\% (3) y en cáncer de pulmón en el $7,1 \%$ (4) aunque asintomáticas. Se presenta un caso de metástasis coroideas sintomáticas simultáneas con metástasis cerebrales en un paciente con cáncer de pulmón y se revisa el diagnóstico diferencial y las opciones terapéuticas de esta localización metastásica.

\section{CASO APORTADO}

Mujer de 73 años diagnosticada de adenocarcinoma pulmonar (T3NOM0) a raíz de un cuadro de disfonía persistente. Se trató quirúrgicamente mediante lobectomía superior derecha, cuyo resulta- 
do histológico fue de adenocarcinoma (pT2N2M0) con márgenes libres. No se realizó ningún tratamiento complementario. A los dos años y diez meses de la intervención quirúrgica, la paciente refirió nuevamente un cuadro de disfonía y fue diagnosticada de recidiva mediastínica y metástasis pulmonares bilaterales. Se inició tratamiento con quimioterapia según esquema carboplatino-gemcitabina por un total de cinco ciclos, obteniendo una respuesta parcial. A las dos semanas de finalizar el último ciclo de quimioterapia la paciente refirió una pérdida de la agudeza visual del ojo izquierdo sin otras alteraciones neurológicas. En la exploración oftalmológica se constató reducción de la agudeza visual en el ojo izquierdo a 4/10. El examen biomicroscópico de polo anterior y la presión intraocular eran normales. En el fondo de ojo, tras dilatación y ciclopléjia, se observó una lesión blanco-amarillenta, de 5 diámetros papilares, subretiniana, en el cuadrante temporal superior que provocaba un discreto desprendimiento de retina exudativo (Fig. 1a). En la angiografía fluoresceínica se demostró una lesión hipercaptante ya en la fase inicial de la prueba (Fig. 1b). En esta fase inicial es fácil distinguir la zona de desprendimiento retiniano exudativo que aparece hipocaptante rodeando la lesión. En tiempos medios de la angiografía, la lesión aparece como intensamente hipercaptante (Fig. 1c) lo cual demuestra la importante vascularización de la lesión. En etapas tardías se aprecia una permanencia de la captación del contraste (Fig. 1d). Estos datos clínicos permitieron el diagnóstico de metástasis coroidea situada en el cuadrante temporal superior del ojo izquierdo. El estudio de extensión mediante tomografía computerizada (TC) craneal y gammagrafía ósea objetivó múltiples metástasis cerebrales y óseas. Inició irradiación holocraneal administrando una dosis total de 20Gy/5 fracciones, constatando una mejoría clínica significativa de la agudeza visual.

\section{DISCUSIÓN}

Las metástasis coroideas de cáncer de pulmón pueden presentarse como primera manifestación clínica de extensión metastásica de la enfermedad (5) o asociadas a metástasis cerebrales (6), aunque suelen presentase cuando existen al menos otras dos localizaciones metastásicas (4) siendo las más frecuentes cerebro (85\%) y hueso $(55 \%)(7)$.

La sintomatología más frecuente en las metástasis coroideas es la disminución de la agudeza visual, fotofobia, miodesopsias y escotomas, perceptibles por el paciente o apreciables en la campimetría (8). Sin embargo, entre un 1-20\% de las metástasis cerebrales, también pueden presentar diferentes alteraciones visuales en función de la zona de la vía óptica en la que se asiente la lesión. Ante un paciente diagnosticado de neoplasia de pulmón que presente alteraciones visuales, debe plantearse el diagnóstico diferencial entre metástasis cerebral y/o coroidea. Si las alteraciones visuales se acompañan de focalidad neurológica o signos de hipertensión intracraneal, habrá que sospechar metástasis cerebrales, que se deberá confirmar mediante TC craneal. Si por el contrario no existe clínica neurológica acompañante, se requerirá examen oftalmológico para la detección de la posible metástasis coroidea y estudio de extensión mediante TC craneal debido a la alta probabilidad de asociación.

En los pacientes asintomáticos el tratamiento más recomendable está por determinar, ya que no se han descrito estudios en los que se haya valorado esta situación. En general se

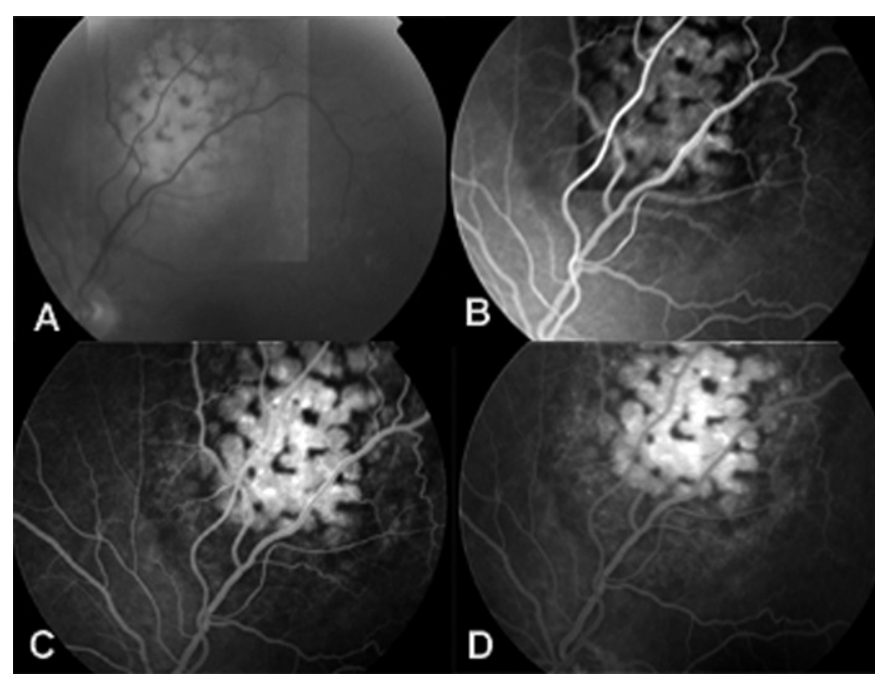

Fig. 1. (A) Retinografía. Lesión en cuadrante temporal superior, subretiniana, desprendimiento de retina exudativo circundante. (B) Fase inicial de la angiografía fluoresceínica. La lesión muestra una gran apetencia por el contraste. La zona hipocaptante perilesional pone de manifiesto el desprendimiento de retina exudativo circundante. (C) En fases medias de la angiografía la lesión se muestra intensamente hipercaptante. (D) En las fases tardías de la angiografía la lesión muestra retención de contraste y aparece leve captación de contraste del desprendimiento de retina exudativo.

suele optar por la observación o bien plantear una "paliación profiláctica" en función del tipo de tumor primario y de las expectativas de vida. El tratamiento de elección en los pacientes sintomáticos consiste en administrar radioterapia. Rosset y cols (9) en un estudio retrospectivo de 78 lesiones, y con dosis de irradiación entre 20-53 Gy/10-30 fracciones, obtuvo hasta un $90 \%$ de respuestas completas y parciales de la agudeza visual con dosis de irradiación superiores a 35,5 Gy. El único estudio prospectivo para evaluar la eficacia de la radioterapia demuestra un $39 \%$ de respuestas completas y un $26 \%$ de parciales (total respuestas 65\%) evaluadas por ultrasonografía, y un $86 \%$ de respuestas clínicas de la agudeza visual en pacientes sintomáticos, administrando una dosis total de 40 Gy/20 fracciones (2).

La expectancia de vida de los pacientes con cáncer de pulmón metastásico con metástasis coroideas es corta (inferior a seis meses) por lo que un programa de detección específico tiene un beneficio muy limitado, especialmente por las características asintomáticas de esta situación clínica. Sin embargo ante la sospecha clínica de metástasis coroideas es recomendable efectuar una exploración oftalmológica para establecer el diagnóstico y una TC craneal, ya que la presencia de metástasis cerebrales modificaría el tratamiento en cuanto al volumen y técnica de irradiación. Como ya se ha comentado, la expectancia de vida de los pacientes con cáncer de pulmón metastático es corta. Aún así, mediante un diagnóstico y tratamiento adecuados de las metástasis coroideas, puede mejorar de forma significativa la calidad de vida de estos pacientes. 


\section{Bibliografía}

1. Albert DM, Rubenstein RA, Scheie HG. Tumor metastasis of the eye. Part I. Incidence in 213 adult patients with generalized malignancy. Am J Ophthalmol 1963; 63: 723-69.

2. Wiegel T, Bottke D, Kreusel KM, Schmidt S, Bornfeld N, Foerster MH, Hinkelbein W. External beam radiotherapy of choroidal metastasesfinal results of a prospective study of the German Cancer Society (ARO 95-08). Radiother Oncol 2002; 64: 13-83.

3. Wiegel T, Kreusel KM, Bornfeld N, Bottke D, Stange M, Foerster MH, Hinkelbein W. Frequency of asymptomatic choroidal metastasis in patients with disseminated breast cancer- results of a prospective screening programme. Br J Ophthalmol 1998; 82: 1159-61.

4. Kreusel KM, Wiegel T, Stange M, Bornfeld N, Hinkelbein W, Foerster MH. Choroidal metastases in disseminated lung cancer: Frequency and risk factors. Am J Ophthalmol 2002; 134: 445-7.
5. Kreusel KM, Bornfeld N, Hosten N, Wiegel T, Foerster MH,. Solitary choroidal metastasis as the first sign of metastatic lung carcinoid. Arch Ophthalmol 1998; 116: 1396-7.

6. Shields JA, Pérez N, Shields CL, Foxman S, Foxman B. Simultaneous choroidal and brain metastasis as initial manifestations of lung cancer. Ophthalmic Surg Lasers 2002; 33: 323-5.

7. Kreusel KM, Bornfeld N, Hosten N, Wiegel T, Foerster MH. Solitary choroidal metastasis as the first sign of metastatic lung carcinoid. Arch Ophthalmol 1998; 116: 1396-7.

8. Shields CL, Shields JA, Gross NE, Schwartz GP, Lallay SE. Survey of 520 eyes with uveal metastases. Ophthalmology 1997; 104: 126576.

9. Rosset A, Zografos L, Coucke P, Monney M, Mirimanoff RO. Radiotherapy of choroidal metastases. Radiother Oncol 1998; 46: 263-8. 